\title{
Somatostatin Analogues Suppress the Inflammatory Reaction In Vivo
}

\author{
Katia Karalis, " George Mastorakos, * George P. Chrousos, * and George Tolis \\ *Developmental Endocrinology Branch, National Institute of Child Health and Human Development, National Institutes of Health, \\ Bethesda, Maryland 20892; and the ${ }^{\ddagger}$ Department of Endocrinology, Hippokration Hospital, Athens University Medical School, Athens \\ 11527, Greece
}

\begin{abstract}
Somatostatin (Sms) and its agonist analogues inhibit the secretory activities of endocrine and neural cells. Recent studies have suggested that Sms has significant immunomodulatory properties. In this study, we examined the effects of two Sms octapeptide analogues on the inflammatory reaction in vivo. BIM 23014 (Somatulin) and Sandostatin were administered to male Sprague-Dawley rats subject to carrageenin-induced aseptic inflammation, at doses of $2-10 \mu \mathrm{g} / \mathrm{rat}$, given either systemically or locally. Animals were killed $7 \mathrm{~h}$ after the induction of the inflammation, and the inflammatory exudates were aspirated and quantitated in terms of volume and leukocyte concentration. Sms analogues, administered via either route, significantly reduced the volume and the leukocyte concentration of the exudate in a time-and dose-dependent fashion. In corroboration of these, immunohistochemical evaluation of the levels of local inflammatory mediators, such as immunoreactive (Ir) TNF- $\alpha$, Irsubstance $\mathbf{P}$, and Ircorticotropin-releasing hormone, was inhibited significantly by Sms analogue treatment. These findings suggest that Sms analogues have significant antiinflammatory effects in vivo, associated with suppression of proinflammatory cytokines and neuropeptides. Furthermore, these data suggest that Sms agonists may be useful in the control of inflammatory reaction. (J. Clin. Invest. 1994. 93:20002006.) Key words: somatostatin $•$ inflammation - tumor necrosis factor- $\alpha \bullet$ substance $P \bullet$ corticotropin-releasing hormone
\end{abstract}

\section{Introduction}

Somatostatin (Sms) ${ }^{1}$ and its analogues exert inhibitory actions on the secretory activities of neurons and neuroendocrine cells $(1,2)$. In the dorsal root ganglia, a distinct population of neurons with small dark perikarya and unmyelinated axons is rich in Sms (3). The latter is transported from these sites to peripheral sensory nerve terminals, from which it is released in response to noxious stimuli (2), involved thus in the axon reflex to local injurious stimuli. In addition, Sms is present in the spinal cord and the substantia gelatinosa (4), both areas in-

Address correspondence to George P. Chrousos, M.D., National Institutes of Health, National Institute of Child Health and Human Development, Developmental Endocrinology Branch, Building 10, Room 10N244, 9000 Rockville Pike, Bethesda, MD 20892, or Katia Karalis, M. D., Children's Hospital, Division of Endocrinology, 300 Longwood Avenue, 416 Enders, Boston, MA 02115.

Received for publication 23 August 1993 and in revised form 13 January 1994.

1. Abbreviations used in this paper: $\mathrm{CRH}$, corticotropin-releasing hormone; Ir, immunoreactive; r/h, rat/human; sc, subcutaneous; Sms, somatostatin; sP, substance $P$.

The Journal of Clinical Investigation, Inc.

Volume 93, May 1994, 2000-2006 volved in transmission of nociception (5). Sms analogues exert analgesic effects when administered systemically, locally, epidurally, or intrathecally (6-10). It has been proposed that many of the actions of Sms released from the peripheral nervous system are in opposition to those of substance $P(s P)$, a major mediator of pain and neurogenic inflammation (11).

Receptors for Sms have been identified on mononuclear leukocytes (12) and on T lymphocytes (13). Sms suppresses endotoxin-induced leukocytosis, release of colony-stimulating activity by splenic lymphocytes, and immunoglobulin synthesis (14). Sms and SP exert opposite effects upon T lymphocytes, respectively inhibiting and stimulating their activity (15, 16). A decrease of peripheral lymphocyte number and function were reported in a patient with a somatostatinoma, as well as in normal controls given Sms-14 (17). These findings suggested that Sms may have immunoregulatory properties and that it might act as an antiinflammatory agent.

The purpose of this study was to examine the potential antiinflammatory actions of two Sms analogues $(18,19)$ in vivo in an established rat model of acute inflammation. In addition to examining gross indices of the inflammatory response, such as volume and cellularity of the inflammatory exudate, the local presence of immunoreactive (Ir) Sms and the ability of Sms analogues to modify the local expression of known mediators of inflammation, such as TNF- $\alpha$ (20), sP (21), and corticotropin-releasing hormone (CRH) (22), were studied.

\section{Methods}

\section{Animals}

Male Sprague-Dawley rats weighing 150-200 g were purchased from Taconic Farms Inc. (Germantown, NY). Rats were maintained in a standard light-dark schedule (lights on from 7 am to $7 \mathrm{pm}$ ), fed standard rat chow ad lib., and had free access to water.

\section{Materials}

Carrageenin, cyanogen bromide-activated Sepharose 4B, and 3,3-diaminobenzidine tetrahydrochloride were obtained from Sigma Chemical Co. (St Louis, MO); synthetic rat/human (r/h) CRH 1-41 from Peninsula Laboratories, Inc. (Belmont, CA); 10\% formalin (Formalde-Fresh) from Fischer Scientific Co. (Pittsburgh, PA); Vectastain ABC kits from Vector Laboratories, Inc. (Burlingame, CA); rabbit IgG from Jackson ImmunoResearch Laboratories, Inc. (West Grove, PA); and light green SF from Roboz Surgical Instrument Co., Inc. (Washington, DC). The Sms analogue BIM 23014 (Somatulin) was a gift of IPSEN International (Paris, France), while the Sms analogue Sandostatin was purchased commercially (Sandoz, Inc., East Hanover, NJ).

\section{Induction of subcutaneous carrageenin inflammation}

Experimental inflammation was induced by injection of carrageenin in previously generated subcutaneous (sc) air pouches in the intrascapular area of the rats as described by Tsurufuji (23) and modified slightly by us (24). $4 \mathrm{ml}$ of $2 \%$ carrageenin solution in normal saline was injected into the 24 -h previously formed sc air pouch. The animals were killed $7 \mathrm{~h}$ after the carrageenin administration, the time of peak inflam- 
mation, and the exudates were aspirated and evaluated in terms of volume and leukocyte concentration. The leukocytes in the inflammatory exudate were mainly PMNs attracted to the local inflammatory site from the general circulation (25).

\section{Experimental protocol}

Rats were divided in groups of eight and given carrageenin and concomitantly either an intraperitoneal (i.p./systemic administration) or an intrapouch (local administration) injection of $1 \mathrm{ml}$ of either BIM 23014 or Sandostatin solutions in $0.9 \% \mathrm{NaCl}$ at increasing doses $(0-10$ $\mu \mathrm{g} / \mathrm{rat})$.

\section{Tissue preparation}

Pieces of inflammatory granuloma from the pouch were dissected en bloc with the surrounding tissues immediately after killing. These tissue specimens were fixed in 10\% formalin and embedded in paraffin.

\section{Immunohistochemistry}

Peptide antisera production and purification. Polyclonal antisera against Sms (GC-27), r/hCRH (TS-2), TNF- $\alpha$, and sP were produced in rabbits, as described previously $(22,26)$. Before use they were affinity-purified by adsorption to synthetic Sms 1-14, r/hCRH 1-41, TNF$\alpha$, and sP, respectively, coupled to cyanogen bromide-activated Sepharose 4B. Briefly, the antisera were added to the Sms-, r/hCRH-, TNF$\alpha$ - and sP-Sepharose 4B conjugates ( $1 \mathrm{mg}$ of Sms 1-14, r/hCRH 1-41, TNF- $\alpha$, or sP on $200 \mu \mathrm{g}$ gel), respectively, and incubated at room temperature for $2 \mathrm{~h}$. These suspensions were packed in $4 \times 0.7-\mathrm{cm}$ columns and washed with phosphate buffer $(20 \mathrm{mM}$ sodium phosphate, $0.5 \mathrm{M}$ sodium chloride, $\mathrm{pH}$ 7.3) until the $\mathrm{OD}_{280}$ returned to baseline. The elutions obtained from these washings were depleted of anti-Sms, anti-r/hCRH, anti-TNF- $\alpha$, and anti-sP IgG, respectively. The antibodies bound to the affinity columns (affinity-positive IgG fractions) were eluted with thiocyanate buffer ( $3 \mathrm{M}$ potassium thiocyanate, $0.5 \mathrm{M}$ ammonium hydroxide) and dialyzed against several changes of PBS.

Immunohistochemistry. Tissue specimens were preserved in $10 \%$ formalin. They were embedded in paraffin and sectioned onto gelatincoated microscope slides at a thickness of $6 \mu \mathrm{m}$. Immunoperoxidase staining was performed with the Vectastain $\mathrm{ABC}$ kit using the manufacturer's suggested protocol and reagents $(27,28)$. All subsequent procedures took place at room temperature. The sections were deparaffinized with two 5-min washes in xylene and rehydrated by sequential rinses in absolute, $90 \%, 80 \%$, and $70 \%$ ethanol. Endogenous peroxidase activity was exhausted by incubation with $0.3 \%$ peroxide in methanol for $45 \mathrm{~min}$. The sections were then incubated sequentially with $0.1 \%$ BSA in PBS for 20 min and with diluted goat serum (1:66.7) for 20 $\mathrm{min}$, and in a humid chamber with the affinity-purified rabbit antibody to $\mathrm{r} / \mathrm{hCRH}(50 \mu \mathrm{g} / \mathrm{ml}$ anti-Sms, anti-r/hCRH, anti-TNF- $\alpha$, or anti$\mathrm{sP})$, the control, affinity-negative IgG fraction $(50 \mu \mathrm{g} / \mathrm{ml})$, or nonimmune normal rabbit IgG $(50 \mu \mathrm{g} / \mathrm{ml})$. After $40 \mathrm{~min}$, the sections were washed with PBS and incubated with biotinylated goat anti-rabbit IgG for $30 \mathrm{~min}$. The sections were washed further with PBS and incubated with avidin and a biotinylated horseradish peroxidase complex for 45 min. Finally, the sections were washed, and color was developed by immersing sections in a solution of $0.05 \% \mathrm{wt} / \mathrm{vol}$ 3,3-diaminobenzidine tetrahydrochloride, $0.04 \% \mathrm{wt} / \mathrm{vol}$ nickel chloride, and $0.01 \%$ hy-

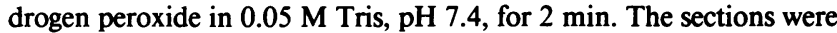
counterstained with $0.5 \%$ light green SF. Positive staining was revealed as black to dark green spots, whereas light green color was seen in Sms-, $\mathrm{r} / \mathrm{hCRH}-, \mathrm{TNF}-\alpha-$, and sP-free areas, affinity-negative control sections, and nonimmune normal rabbit IgG control sections. The intensity and concentration of the specific staining on the tissue specimens were graded by two masked observers on a $0-4+$ scale.

\section{Statistical analysis}

All results are expressed as mean \pm SEM. ANOVA followed by Fischer's least significant difference test was performed. Differences were considered significant when $P<0.05$.

\section{Results}

Immunohistochemical detection of IrSms in sc carrageenin-induced inflammatory granulomas. The presence of IrSms in sc carrageenin-induced inflammatory areas was examined immunohistochemically on tissue sections obtained from the inflammatory areas at the peak of the inflammatory reaction, $7 \mathrm{~h}$ after the injection of carrageenin. IrSms was detected in the cytoplasm of all three types of accessory cells, i.e., macrophages, tissue fibroblasts, and vascular endothelial cells (Fig. 1). No IrSms was detected in the nuchal tissue of noninflamed control animals, with the exception of hair follicles and sweat glands.

Effects of the systemic administration of BIM 23014 and Sandostatin on the carrageenin-induced local aseptic inflammatory reaction. Systemic administration of increasing doses of BIM 23014 or Sandostatin (2-10 $\mu \mathrm{g} / \mathrm{rat})$ concomitantly with carrageenin resulted in a reduction of both the volume and the cell concentration of the inflammatory exudate (Fig. 2). The reduction of the volume ranged between 12 and $45 \%$ lower than control values after BIM 23014 administration and between 11 and $40 \%$ lower than control values after Sandostatin administration. The leukocyte concentrations were $25-40$ and $42-48 \%$ of control values in the BIM 23014- and Sandostatintreated groups, respectively. There was no apparent dose-response for Sandostatin at the dose range applied.

Effects of the local administration of BIM 23014 and Sandostatin on the carrageenin-induced aseptic inflammatory reaction. Direct local (intrapouch) administration of increasing doses $(2-10 \mu \mathrm{g} / \mathrm{rat})$ of BIM 23014 or Sandostatin concomitantly with carrageenin resulted in a reduction of both inflammatory parameters measured (Fig. 3). The volume of the inflammatory exudate was lower by $10-40 \%$ and $<10 \%$ than the control values after BIM 23014 and Sandostatin administration, respectively. The leukocyte concentrations of the exudates were also lower by 5-40 and 55-65\% compared with controls for BIM 23014- and Sandostatin-treated groups, respectively. Both analogues reduced significantly the leukocyte concentrations in the inflammatory exudate, while the volume of the latter was reduced significantly only by BIM 23014 at the dose range used. In addition, there was no apparent dose-response for the antiinflammatory effect of Sandostatin at the doses tested.

Time course of the inflammatory reaction in control and BIM 23014-treated animals. Two groups of 30 animals per group, subject to carrageenin-induced inflammation, received simultaneously intraperitoneal injections of either $10 \mu \mathrm{g} \mathrm{BIM}$ 23014 or normal saline (control group). 10 animals from each group were killed 2,5 , and finally $7 \mathrm{~h}$ after the induction of the inflammation. The degree of the inflammatory reaction was evaluated as described above (Fig. 4). Both the volume and the leukocyte concentration of the exudate increased with time, reaching a zenith at $7 \mathrm{~h}$ in the control group. In the Sms analogue-treated group, both the volume and the leukocyte concentration were significantly lower than those of the control group.

Immunohistochemical detection of IrTNF- $\alpha$, IrsP, and IrCRH in sc carrageenin-induced inflammatory areas before and after systemic administration of BIM 23014. The presence of IrTNF- $\alpha$, IrsP, and IrCRH was examined immunohistochemically on sections obtained from the carrageenin-induced inflammatory areas of the nuchal skin of rats treated with BIM 23014 and on untreated matched control rats, at $0,2,5$, and $7 \mathrm{~h}$ 

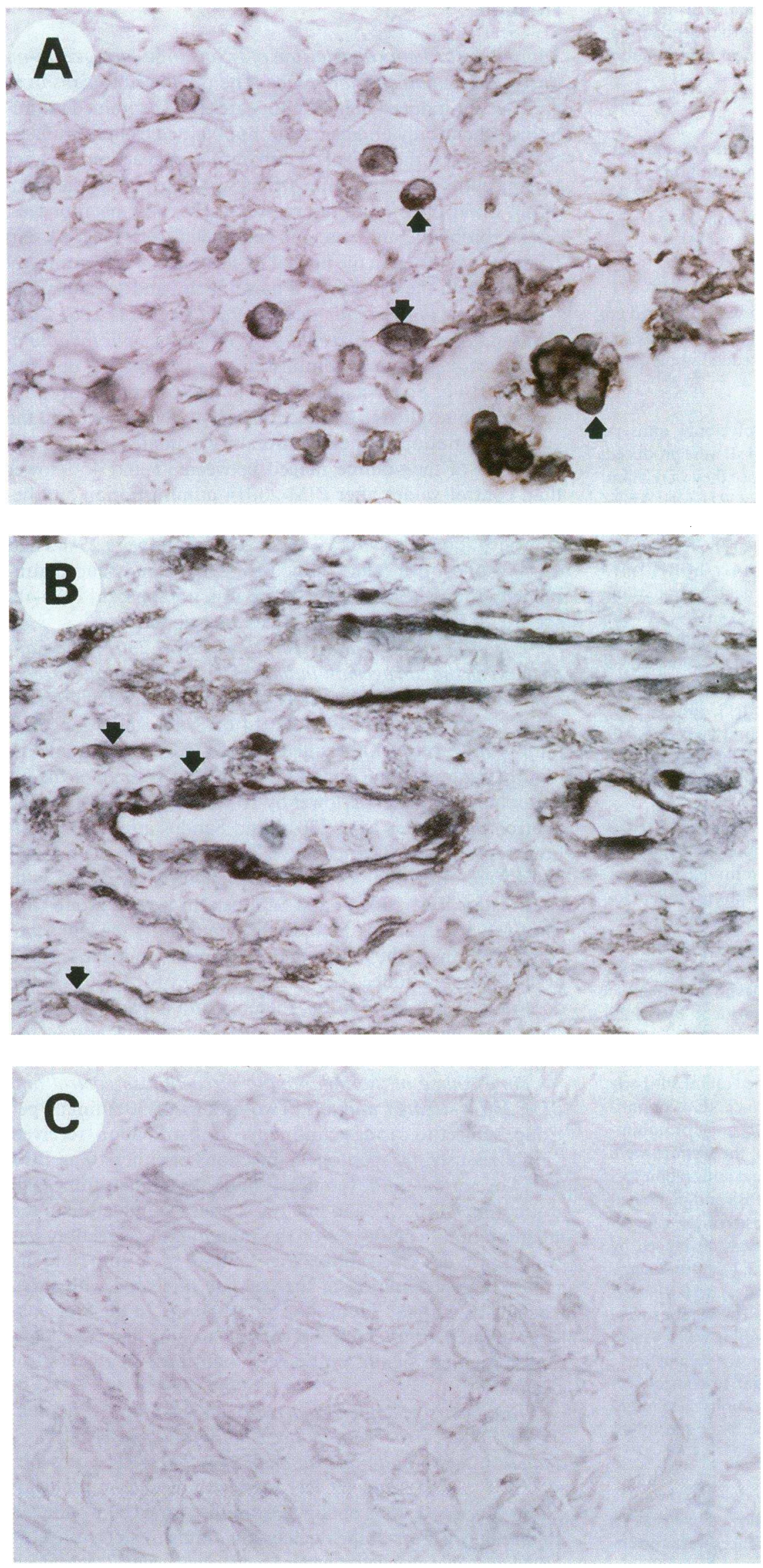

Figure 1. Immunohistochemical localization of IrSms $(A$ and $B)$ on sections obtained from the carrageenin-induced inflammatory areas of the nuchal skin of rats at $7 \mathrm{~h}$ after the induction of the inflammatory reaction. Positive staining is revealed as brown-black spots. Mononuclear inflammatory leukocytes $(A)$, tissue fibroblasts $(B)$, and vascular endothelial cells $(B)$ show intense Sms immunostaining. No specific staining can be seen on the same carrageenin-induced inflammatory areas on control adjacent tissue sections incubated with the affinity-negative IgG fraction of the anti-Sms antiserum $(C) . \times 250$. 

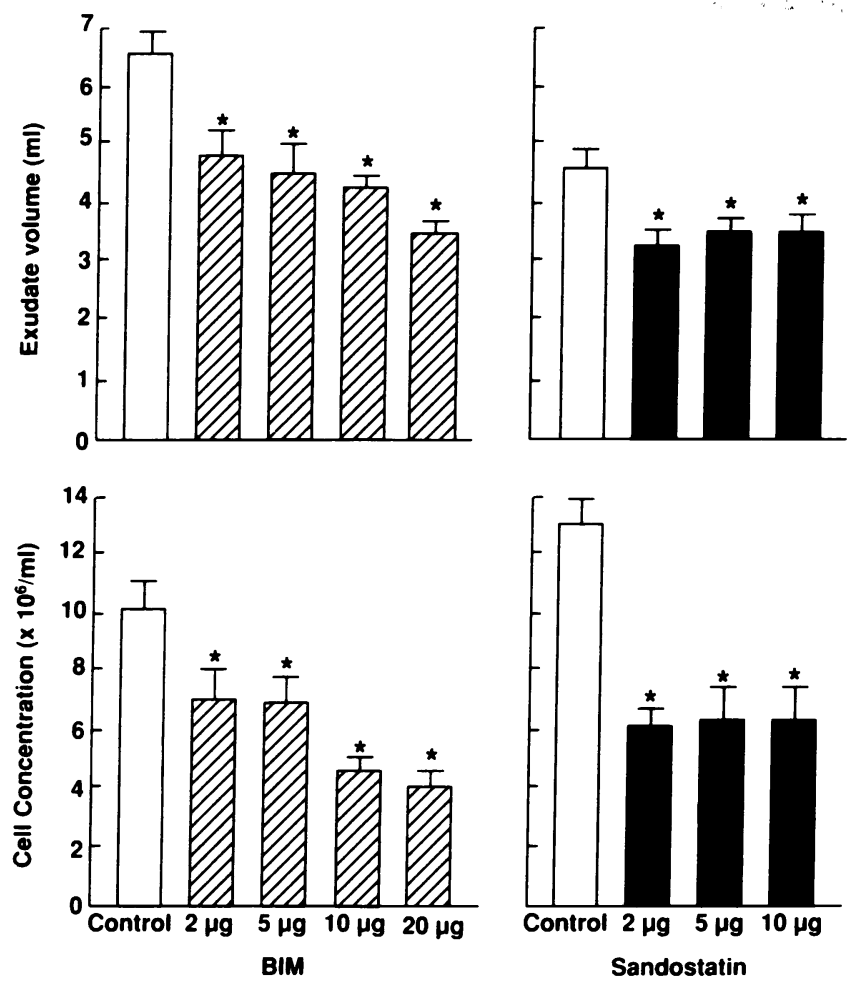

Treatment Groups

Figure 2. Effects of systemic administration of Sms analogues on inflammation. The volume (top) of the exudate was reduced significantly after administration of either BIM 23014 ( hatch bars) or Sandostatin (black bars). The effect of BIM 23014 was dose dependent, while no significant differences were seen between the various doses of Sandostatin used. The leukocytic concentration in the exudates was reduced by administration of either of the analogues (bottom). BIM 23014 administration resulted in a dose-dependent effect, while no significant differences were seen between the various doses of Sandostatin used ( $n=8-10$ rats/group). ${ }^{*}$ Significant difference at 95\% compared with control levels (open bars).

after the induction of the inflammatory reaction. IrTNF- $\alpha$, IrsP, and IrCRH were detected in the cytoplasm of tissue macrophages, tissue fibroblasts, and endothelial cells of the inflammatory sites of the placebo and BIM 23014-treated animals (Fig. 5). Semiquantitative analysis of immunohistochemical staining with a grading system from 0 to $4+$ gave the results represented in Fig. 6. At 7 h, IrTNF- $\alpha$ was $2+$ versus 0 , IrsP $2.5+$ versus $0.5+$, and IrCRH $3+$ versus $1+$, for untreated and BIM 23014-treated animals, respectively.

\section{Discussion}

Sms has been detected not only in the central and peripheral nervous system but, in addition, in several nonneuronal peripheral sites (1-3). Neuronal Sms stored in secretory granules and released via $\mathrm{Ca}^{2+}$-dependent reverse pinocytosis has been thought to act as a neurotransmitter or neuromodulator (29). Sms produced by sensory fibers and/or postganglionic sympathetic neurons $(2,4,30)$ may also act as a modulator of the neurogenic component of inflammation. Capsaicin treatment, which reduces the degree of neurogenic inflammation, also decreases Sms immunoreactivity in the spinal cord, suggesting that this neuropeptide may be involved in the reflex response to
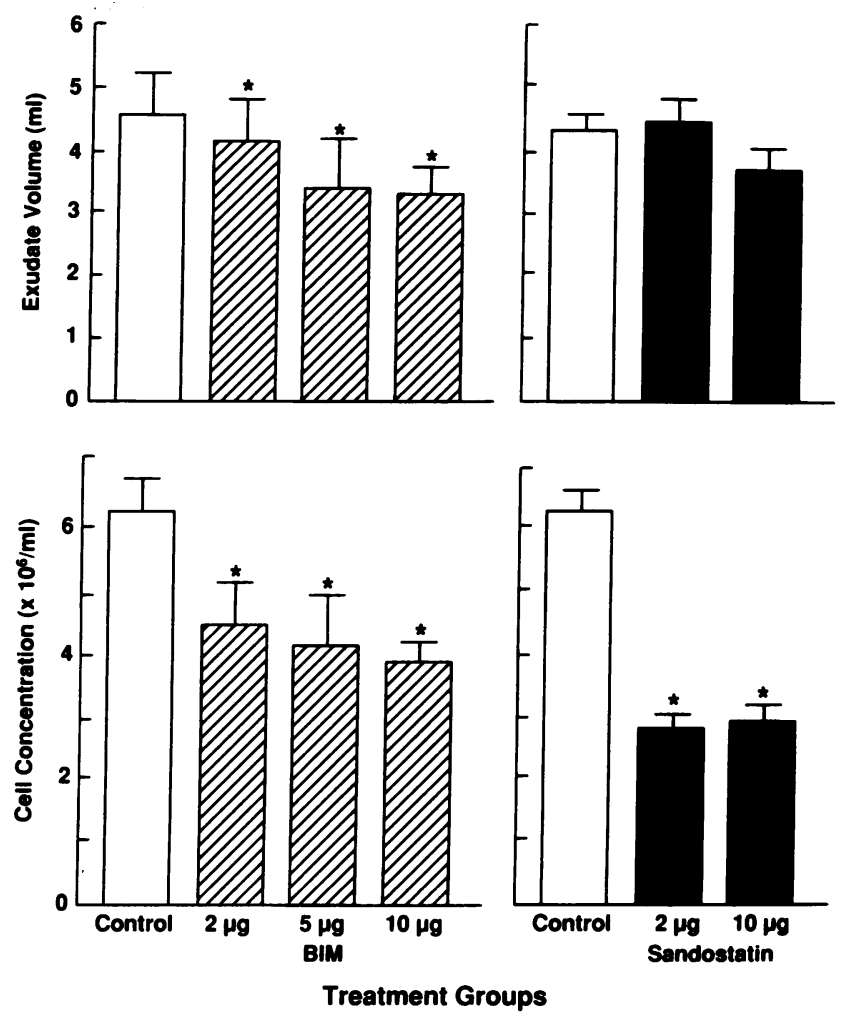

Figure 3. Effects of local administration of Sms analogues on inflammation. The volume of the exudate (top) was reduced after BIM 23014 administration (hatch bars), while Sandostatin (black bars) caused no significant differences. On the contrary, both analogues reduced the leukocyte concentration (bottom), even at the lowest dose used $(2 \mu \mathrm{g})(n=8-10$ rats/group $) .{ }^{*}$ Significant difference at 95\% compared with controls (open bars).

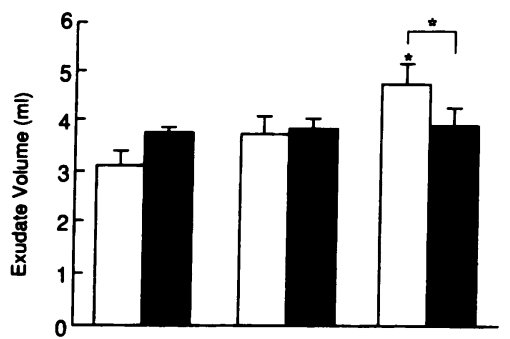

Figure 4. Time course of the inflammatory reaction after systemic administration of $10 \mu \mathrm{g}$ of BIM 23014 (black bars). The volume (top) of the exudate was lower in the BIM 23014treated group than in the control group (open bars) at $7 \mathrm{~h}$. In the ana-

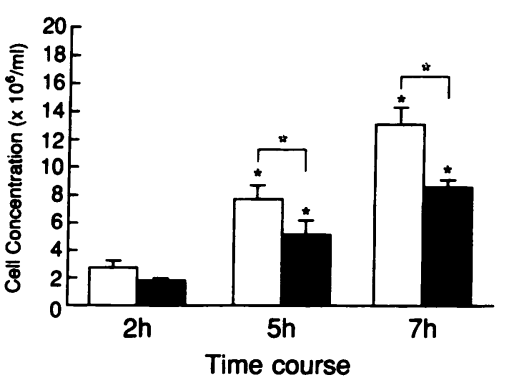

logue-treated group, the volume of the exudate reached its peak at $2 \mathrm{~h}$ and remained constant thereafter, while in the control group it reached its peak at $7 \mathrm{~h}$. The leukocyte concentration (bottom) increased over the 7-h period and was lower in the BIM 23014-treated group than in the control group both at 5 and $7 \mathrm{~h}$ ( $n=8-10$ rats/group). Solid star, significant difference at $95 \%$ compared with values at 2 h. Open star, significant difference at $95 \%$ compared with control values at the same time point. 

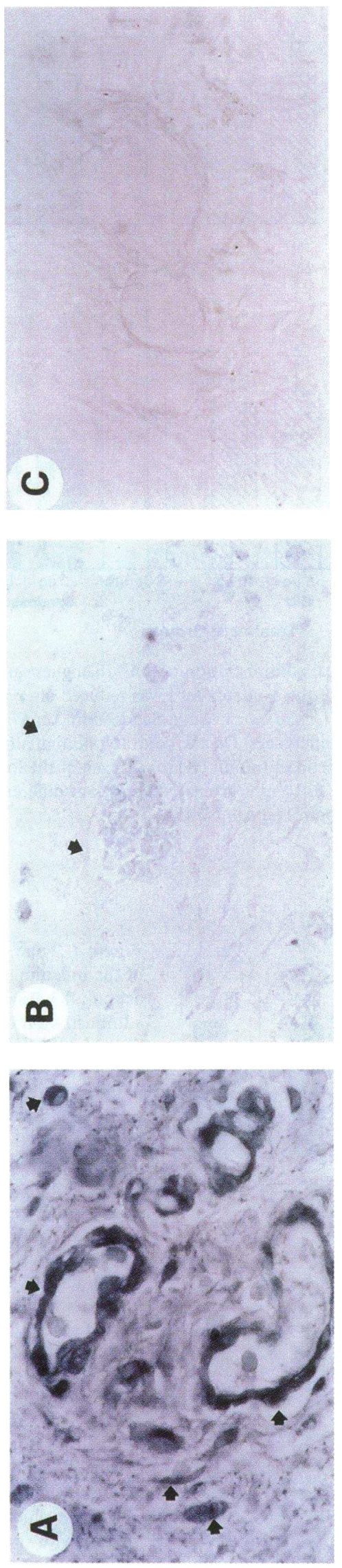
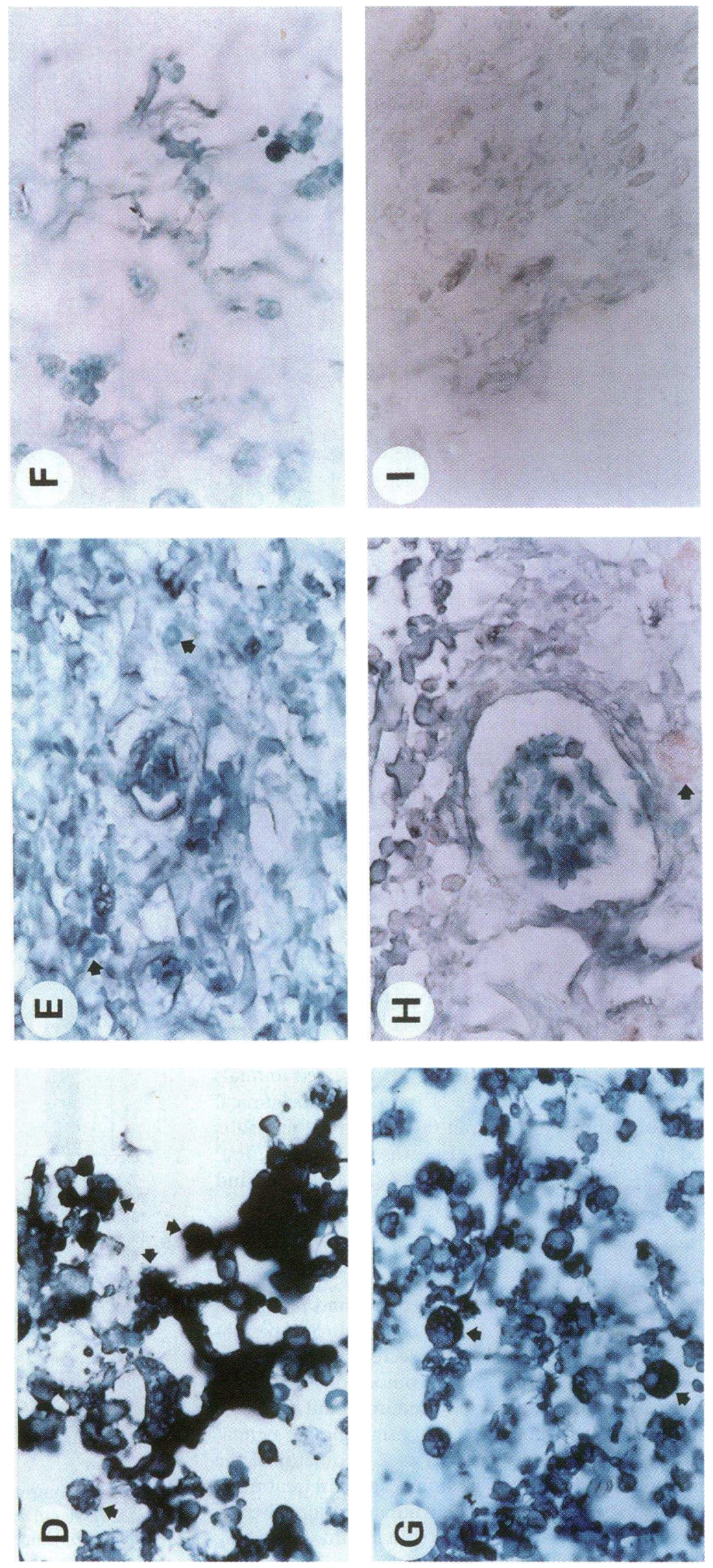

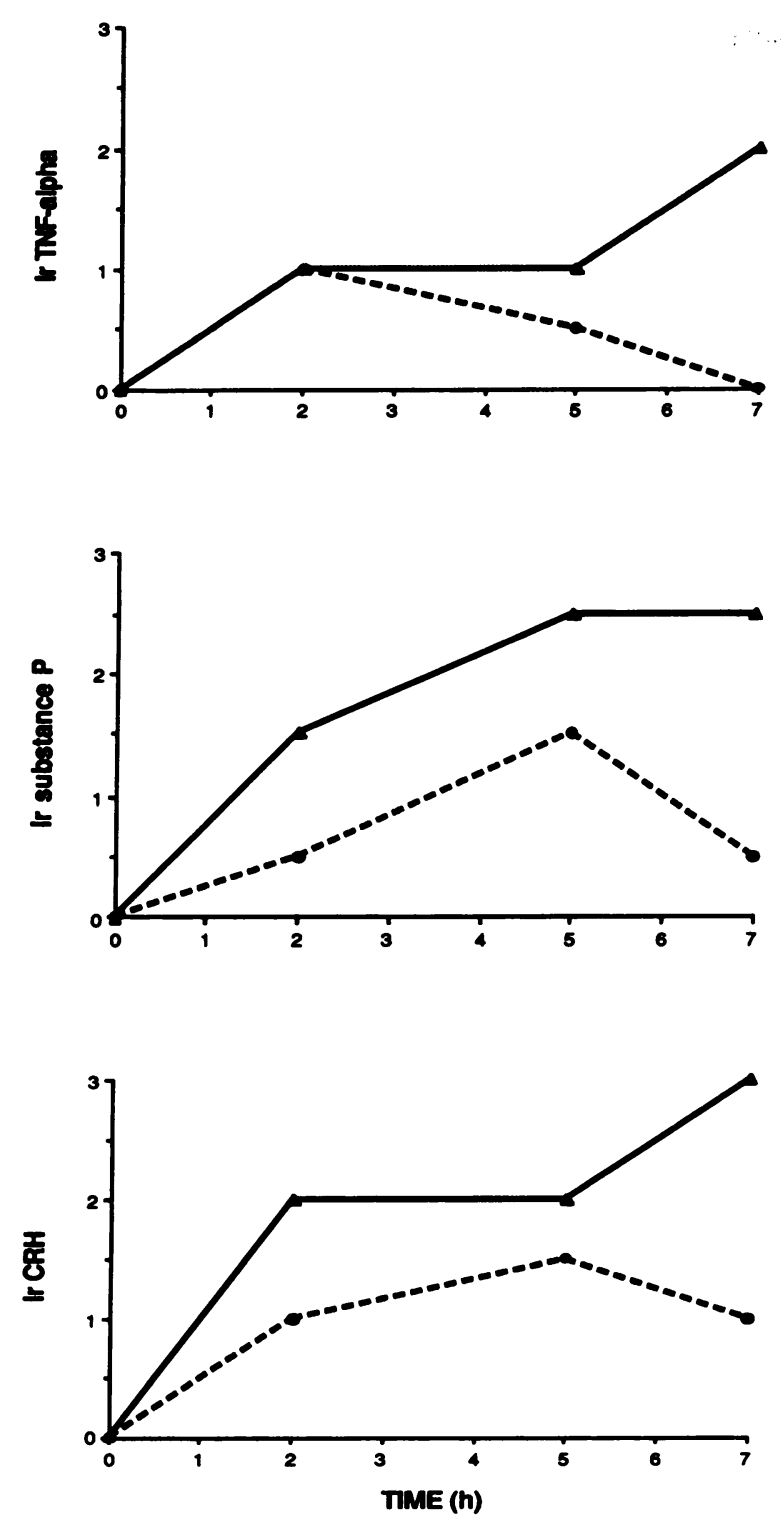

Figure 6. Graphic representation of semiquantitative analysis with a grading system from 0 to $4+$ of TNF- $\alpha$ (top), $\mathrm{sP}$ (middle), and CRH (bottom) immunoreactivity on the carrageenin-induced inflammatory areas of the nuchal skin of rats treated with (dotted line) and without (continuous line) BIM 23014 at $0,2,5$, and $7 \mathrm{~h}$ after the induction of the inflammatory reaction.

chemical pain stimuli (31). We clearly detected IrSms in the carrageenin-induced inflammatory granulomas. This finding could reflect local release of Sms by sensory fibers $C$ and/or postganglionic sympathetic neurons $(2,4,30)$, as part of the active inflammatory process. The positively stained accessory cells cannot be excluded as additional sources of the Sms present in the inflammatory sites, but could also reflect uptake of neuronal Sms by these cells.

The carrageenin-induced subcutaneous aseptic inflammation was suppressed by BIM 23014 in a dose-dependent fashion, whereas the Sandostatin inhibition of inflammation had no apparent dose-response effect at the dose range used. Use of additional dosages of Sandostatin would probably reveal a sigmoidal relationship between dose and effect. Of the two independent parameters of the inflammatory reaction measured, i.e., the volume of the exudate and the leukocyte concentration, the latter was more dramatically suppressed than the former by either analogue. This suggests that Sms exerts preferential effects on the inhibition of the migration of circulating leukocytes to the inflammatory site (predominantly PMN in the inflammatory exudates in our study) rather than on the suppression of the permeability of the capillary endothelium, which normally increases during inflammation. Blockade of the expression of adhesion molecules and/or their receptors by endothelial cells and/or leukocytes and/or inhibition of secretion of chemotactic substances, such as IL-8, components of the complement system, or leukotrienes, could explain the preferential effects of Sms on leukocyte migration. These findings agree with in vitro data showing inhibition of sP-induced PMN chemotaxis by Sandostatin (32) and with the previously observed Sms-induced suppression of impulse transmission of spinal cord neurons excited by noxious stimuli or local iontophoresis of sP. Furthermore, they agree with the fact that Sms analogues inhibit TNF- $\alpha$ release by LPS-stimulated macrophages (33) and with our data from this study demonstrating that Sms analogues gradually attenuate the secretion and/or activity of inflammatory molecules, such as TNF- $\alpha$, a cytokine (20), sP, a tachykinin (21), and CRH, a local proinflammatory neuropeptide (22), from the beginning of the inflammatory process to its 7 -h peak in this model.

The antiinflammatory effect of both Sms analogues used was achieved after both systemic and local administration, suggesting that effective drug concentrations were reached at the site of inflammation regardless of the route of administration. The antiinflammatory effect observed after local administration was less than that after systemic administration. This might be because of additional effects of the latter at a wider area of regulation of the inflammatory reaction, including perhaps the dorsal root ganglia and the spinal cord (34). Increased local enzymatic degradation of Sandostatin might also account for the decreased potency of this analogue, when administered locally, on the inflammatory exudate volume. Regardless of the route of administration, both Sms analogues resulted in similar antiinflammatory activities, as reflected by suppression of leukocyte concentration, suggesting that their potency in suppressing inflammation is parallel to their ability to suppress growth hormone secretion (35). These data suggest that Sms

Figure 5. Immunohistochemical localization of $\operatorname{IrTNF}-\alpha(A$ and $B), \operatorname{IrsP}(D$ and $E)$, and $\operatorname{IrCRH}(G$ and $H$ ) on sections obtained from the carrageenin-induced inflammatory areas of the nuchal skin of rats treated with BIM $23014(B, E$, and $H)$ and of untreated matched control rats $(A, D$, and $G)$ at $7 \mathrm{~h}$ after the induction of the inflammatory reaction. Positive staining is revealed as black to dark green spots, whereas light green color is used to reveal tissue architecture. TNF- $\alpha$, sP, and CRH immunostaining in mononuclear inflammatory leukocytes, tissue fibroblasts, and vascular endothelial cells is reduced considerably on the sections obtained from BIM 23014-treated rats $(B, E$, and $H)$ compared with the intense immunostaining of these cells on the sections obtained from the untreated control rats $(A, D$, and $G)$. No specific staining can be seen on control adjacent tissue sections obtained from the same carrageenin-induced inflammatory areas of the untreated control rats and incubated with the affinity-negative IgG fractions of the anti-TNF- $\alpha(C)$, anti-sP $(F)$, and anti-CRH $(I)$ antisera. The arrows indicate mononuclear inflammatory leukocytes, tissue fibroblasts, and vascular endothelial cells $(A, B, D, E, G$, and $H) . \times 250$. 
agonists given systemically or applied locally might be useful in the control of inflammatory or autoimmune states.

The inflammatory cytokines TNF- $\alpha$, IL- $1 \beta$, and IL- 6 activate the hypothalamic-pituitary-adrenal axis by primarily causing hypothalamic $\mathrm{CRH}$ secretion, which leads to glucocorticoid secretion through ACTH release (36-39). Glucocorticoid secretion then limits the inflammatory reaction by preventing leukocytes from reaching the inflammatory site and by decreasing the secretion and action of various cytokines and lipid mediators of inflammation $(25,40)$. It is not known whether Sms participates in such a physiologic process as an antiinflammatory peptide, and it would be of interest to examine the local secretion of Sms during glucocorticoid-induced suppression of the inflammatory response. In a preliminary report, dexamethasone was shown to inhibit all proinflammatory agents examined in the carrageenin model of inflammation, including TNF- $\alpha, \mathrm{CRH}$, and sP, while it allowed large, gradual increases of local IrSms over the 7-h assay (41). This suggests that Sms may participate in the physiologic control of inflammation, in addition to glucocorticoids.

\section{Acknowledgments}

We would like to thank IPSEN International (Paris, France) for donating the Sms analogue BIM 23014 (Somatulin) used in this study. Also, we would like to thank Dr. Hajime Sano and Dr. Ronald R. Wilder, National Institute of Arthritis and Musculoskeletal and Skin Diseases, National Institutes of Health for invaluable advice.

\section{References}

1. Vale, W., P. Brazeau, C. Rivier, M. Brown, B. Boss, J. Rivier, R. Burgus, N. Ling, and R. Guillemin. 1975. Somatostatin. Recent Prog. Horm. Res. 31:365397.

2. Reichlin, S. 1983. Somatostatin. N. Engl. J. Med. 309:1495-1501.

3. McCann, S. M. 1982. Physiology and pharmacology of LHRH and somatostatin. Annu. Rev. Pharmacol. Toxicol. 22:491-515.

4. Hokfelt, T., O. Johansson, A. Ljungdahl, J. M. Lundberg, and M. Schultzberg. 1980. Peptidergic neurones. Nature (Lond.). 284:515-521.

5. Kessler, J. A., and I. B. Black. 1981. Similarities in development of substance $P$ and somatostatin in peripheral sensory neurons: effects of capsaicin and nerve growth factor. Proc. Natl. Acad. Sci. USA. 78:4644-4647.

6. Chan, A. W., G. Williams, I. A. MacFarlane, and D. Bowsher. 1991. Octreotide, a long-acting somatostatin analog in diabetic neuropathic pain. Horm. Res. (Basel). 36:86-87.

7. Recht, L. D., and G. M. Abrams. 1986. Neuropeptides and their role in nociception and analgesia. Neurologic Clinics of North America. 4:833-852.

8. Caleri, D., S. Marabini, A. Pancones, and U. Pietrini. 1987. A pharmacological approach to the analgesizing mechanism of somatostatin in cluster headache. Ric. Clin. Lab. 17:155-162.

9. Chrubasik, J. 1985. Somatostatin. Eine Ubersich. Anästhesie Intensivetherapie Notfallmedizin. 20:165-170.

10. Tolis, G., A. Vadalouka, and P. Zourlas. 1989. Postoperative analgesis via a somatostatin analogue Sms 201-995. The Endocrine Society, 71 st Annual Meeting, Seattle. Abstract 865.

11. Gazelius, B., E. Brodin, L. Olgart, and P. Panopoulos. 1981. Evidence that substance $\mathbf{P}$ is a mediator of antidromic vasodilation using somatostatin as a release inhibitor. Acta Physiol. Scand. 113:155-159.

12. Bhathena, S. J., J. Louie, G. P. Schechter, R. S. Redman, L. Wahl, and L. Recant. 1981. Identification of human mononuclear leukocytes bearing receptors for somatostatin and glucagon. Diabetes. 30:127-131.

13. Sreedharan, S. P., K. T. Kodama, K. E. Peterson, and E. J. Goetzl. 1989. Distinct subsets of somatostatin receptors on cultured human lymphocytes. $J$. Biol. Chem. 264:949-952.

14. Hinterberger, W., C. Cerny, M. Kinast, H. Pointner, and K. H. Trag. 1977. Somatostatin reduces the release of colony-stimulating activity (CSA) from PHA-activated mouse spleen lymphocytes. Experientia (Basel). 34:860-862.

15. Wagner, H., K. Hengst, E. Zierden, and U. Gerlach. 1978. Investigations of the antiproliferative effect of somatostatin in man and rats. Metab. Clin. Exp. 27:1381-1386.

16. Payan, D. G., and E. J. Goetzl. 1985. Modulation of lymphocyte function by sensory neuropeptides. $J$. Immunol. $135: 783 \mathrm{~s}-786 \mathrm{~s}$.

17. Vehmeyer, K., P. Schuff-Werner, and G. A. Nagel. 1986. Suppressed colony formation of peripheral blood lymphocytes in a patient with a somatostatinoma. Clin. Immunol. Immunopathol. 41:290-294.

18. Bogden, A. E., J. E. Taylor, J. P. Moreau, D. H. Coy, and D. J. LePage. 1990. Response of human lung tumor xenografts to treatment with a somatostatin analogue (somatuline). Cancer Res. 50:4360-4365.

19. Bauer, W., U. Briner, W. Doepfner, R. Haller, R. Huguenin, P. Marbach, T. J. Petcher, and J. Pless. 1982. SMS 201-995: a very potent and selective octapeptide analog of somatostatin with prolonged action. Life Sci. 31:1133-1141.

20. Beutler, B. A., I. W. Milsark, and A. Cerami. 1985. Cachectin/tumor necrosis factor: production, distribution and metabolic fate in vivo. J. Immunol. 135:3972-3977.

21. Payan, D. G. 1989. Neuropeptides and inflammation: the role of substance P. Annu. Rev. Med. 40:341-352.

22. Karalis, K., H. Sano, J. Redwine, S. Listwak, R. L. Wilder, and G. P. Chrousos. 1991. Autocrine or paracrine inflammatory actions of corticotropinreleasing hormone in vivo. Science (Wash. DC). 254:421-423.

23. Tsurufuji, S., H. Sato, K. R. Min, and K. Ohuchi. 1978. Difference in the antiinflammatory effect of indomethacin between acute and chronic stages of carrageenin-induced inflammation. J. Pharmacobio. Dyn. 1:8-14.

24. Laue, L., S. Kawai, D. D. Brandon, D. Brightwell, K. Barnes, R. A. Knazek, D. L. Loriaux, and G. P. Chrousos. 1988. Receptor-mediated effects of glucocorticoids on inflammation: enhancement of the inflammatory response with a glucocorticoid antagonist. J. Steroid Biochem. 29:591-598.

25. Boumpas, D. T., G. P. Chrousos, R. L. Wilder, T. R. Cupps, and J. E Balow. 1993. Glucocorticoid therapy for immune-mediated diseases: basic and clinical correlates. Ann. Intern. Med. 119:1198-1208.

26. Schürmeyer, T. H., P. C. Avgerinos, P. W. Gold, W. T. Gallucci, T. P. Tomai, G. B. Cutler, Jr., D. L. Loriaux, and G. P. Chrousos. 1984. Human corticotropin-releasing factor in man: pharmacokinetic properties and dose-response of plasma adrenocorticotropin and cortisol secretion. J. Clin. Endocrinol. \& Metab. 59:1103-1108.

27. Hsu, S. M., L. Raine, and H. Fanger. 1981. A comparative study of the peroxidase-antiperoxidase method and an avidin-biotin complex method for studying polypeptide hormones with radioimmunoassay antibodies. Am. J. Clin. Pathol. 75:734-738.

28. Hsu, S. M., L. Raine, and H. Fanger. 1981. Use of avidin-biotin-peroxidase complex $(\mathrm{ABC})$ in immunoperoxidase techniques: a comparison between ABC and unlabeled antibody (PAP) procedures. J. Histochem. Cytochem. 29:577-580.

29. Goetzl, E. J., T. Chernov, F. Renold, and D. G. Payan. 1985. Neuropeptide regulation of the expression of immediate hypersensitivity. J. Immunol. 135:802s-805s.

30. Hökfelt, T., L. G. Elfvin, R. Elde, M. Schultzberg, M. Goldstein, and R. Luft. 1977. Occurrence of somatostatin-like immunoreactivity in some peripheral sympathetic noradrenergic neurons. Proc. Natl. Acad. Sci. USA. 74:35873591 .

31. Ohno, H., Y. Kuraishi, T. Nanayama, M. Minami, M. Kawamura, and M. Satoh. 1990. Somatostatin is increased in the dorsal root ganglia of adjuvantinflamed rat. Neurosci. Res. 8:179-188.

32. Kolasinski, S. L., K. A. Haines, E. L. Siegel, B. N. Cronstein, and S. B. Abramson. 1992. A somatostatin analog as a selective antagonist of neutrophil activation by substance P. Arthritis Rheum. 35:369-375.

33. Bermudez, L. E., M. Wu, and L. S. Young. 1990. Effect of stress-related hormones on macrophage receptors and response to tumor necrosis factor. Lymphokine Res. 9:137-145.

34. Lynn, B., and S. P. Hunt. 1984. Afferent C-fibres: physiological and biochemical correlations. Trends Neurosci. 7:186-188.

35. Sassolas, G., A. G. Harris, A. Deidier-James, and the French Sms 201-995 Acromegaly Study Group. 1990. Long-term effect of incremental doses of the somatostatin analog Sms 201-995 in 58 acromegalic patients. J. Clin. Endocrinol. \& Metab. 71:391-397.

36. Bernardini, R., T. C. Kamilaris, A. E. Calogero, E. O. Johnson, P. W. Gold, and G. P. Chrousos. 1990. Interactions between tumor necrosis factor-alpha, hypothalamic corticotropin-releasing hormone and adrenocorticotropin secretion in the rat. Endocrinology. 126:2876-2881.

37. Sapolsky, R., C. Rivier, G. Yamamoto, P. Plotsky, and W. Vale. 1987. Interleukin 1 stimulates the secretion of hypothalamic corticotropin releasing factor. Science (Wash. DC). 238:522-524.

38. Naitoh, Y., J. Fukata, T. Tominaga, Y. Nakai, S. Tamai, K. Mori, and H. Imura. 1988. Interleukin-6 stimulates the secretion of adrenocorticotropic hormone in conscious, freely moving rats. Biochem. Biophys. Res. Commun. 155:1459-1463.

39. Mastorakos, G., G. P. Chrousos, and J. S. Weber. 1993. Recombinant interleukin 6 activates the hypothalamic-pituitary-adrenal axis in humans. $J$. Clin. Endocrinol. \& Metab. 77:1690-1694.

40. Flower, R., R. Gryglewski, K. Herbaczynska-Cedro, and J. R. Vane. 1972. Effects of anti-inflammatory drugs on prostaglandin biosynthesis. Nat. (Lond.) New Biol. 238:104-106.

41. Karalis, K., G. Mastorakos, H. Sano, J. Redwine, and R. Wilder. 1992. Neuropeptide and cytokine interplay in the regulation of the inflammatory response in a rat experimental model. The Endocrine Society, 74th Annual Meeting, San Antonio, TX. Abstract 1558. 